\title{
Kasuistiken
}

Notfall Rettungsmed 2021 $24: 1114-1118$ https://doi.org/10.1007/s10049-020-00798-1 Angenommen: 25. September 2020 Online publiziert: 6 . November 2020 (c) Springer Medizin Verlag $\mathrm{GmbH}$, ein Teil von Springer Nature 2020

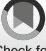

A. Schellhaaß $\beta^{1,2} \cdot$ S. Pöselt ${ }^{2,3} \cdot$ J. Schwietring ${ }^{4} \cdot$ J. Horter ${ }^{2,5} \cdot$ M. Münzberg ${ }^{2,5}$

${ }^{1}$ Klinik für Anästhesie, Intensivmedizin, Schmerztherapie / OP-Abteilung, BG Klinik Ludwigshafen, Ludwigshafen, Deutschland

${ }^{2}$ ADAC-Luftrettungsstation Christoph 5/Christoph 112 Ludwigshafen, Ludwigshafen, Deutschland ${ }^{3}$ DRK Rettungsdienst Vorderpfalz GmbH, Ludwigshafen, Deutschland

${ }^{4}$ Fachbereich Medizin (LML), ADAC Luftrettung gGmbH, München, Deutschland

${ }^{5}$ Abteilung für interdisziplinäre Notfall- und Rettungsmedizin (AiR/N), BG Klinik Ludwigshafen, Ludwigshafen, Deutschland

\section{Luftgebundene Intensivverlegung in Bauchlage bei COVID-19-ARDS}

\section{Situation}

Die diensthabende ITH-Crew wird über die integrierte Rettungsleitstelle mit der Durchführung eines Sekundärtransports einer beatmeten Intensivpatientin mit COVID-19-Pneumonie und schwerem ARDS beauftragt. Im zur Transportplanung telefonisch durchgeführten ArztArzt-Gespräch wird mitgeteilt, dass die Verlegung grundsätzlich aus Kapazitätsgründen erforderlich ist. Darüber hinaus soll die Patientin in ein Krankenhaus mit ECMO-Option verlegt werden, die im abgebenden Krankenhaus nicht zur Verfügung steht. Im weiteren Gespräch ergeben sich keine Aspekte, die nach Einschätzung des diensthabenden Notarztes einem ITH-Transport ent-

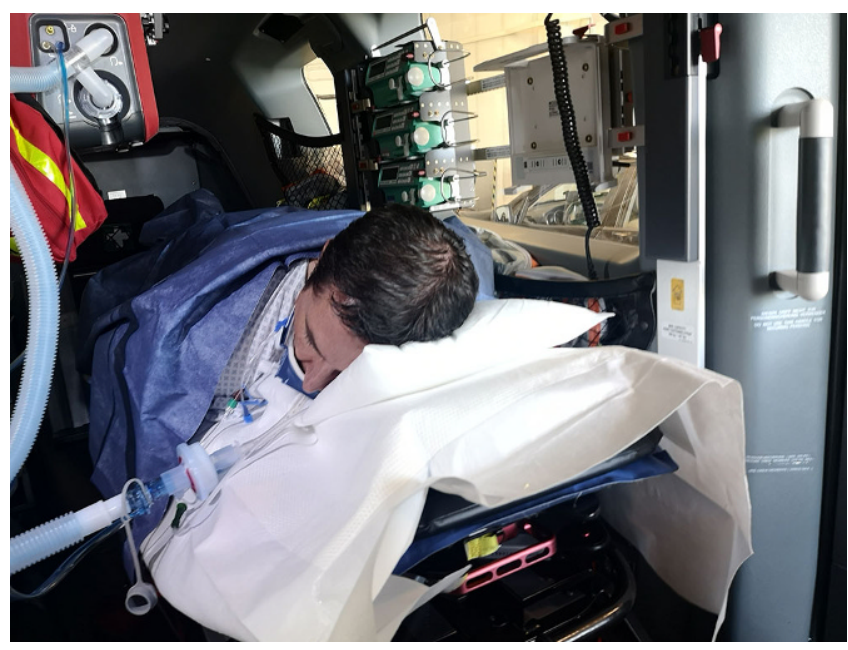

Abb. $1<$ Beatmeter Patient in Bauchlage im ITH (nachgestellt) gegenstehen könnten. Nach Angaben des abgebenden Krankenhauses befindet sich die Patientin in Rückenlage. Die Entfernung vom Stationierungsort des ITH zur abgebenden Klinik beträgt circa $50 \mathrm{~km}$ Luftlinie und von dort zur Zielklinik circa $60 \mathrm{~km}$ Luftlinie. Beim eingesetzten Hubschrauber handelt es sich um eine H145 von Airbus Helicopters, die mittlerweile als Standardmuster für den luftgebundenen Intensivtransport in Deutschland gelten kann. Die ITH-Besatzung besteht neben dem Piloten aus einem Facharzt für Anästhesie mit den Zusatzbezeichnungen Intensiv- und Notfallmedizin und einem HEMS-TC/Notfallsanitäter. Alle Besatzungsmitglieder verfügen über langjäh-

\begin{tabular}{|c|c|}
\hline \multicolumn{2}{|c|}{ Abkürzungen } \\
\hline ARDS & $\begin{array}{l}\text { "Acute respiratory distress } \\
\text { syndrome" }\end{array}$ \\
\hline$B L$ & Bauchlage \\
\hline COVID-19 & "Coronavirus disease 2019" \\
\hline$C R P$ & C-reaktives Protein \\
\hline ECMO & $\begin{array}{l}\text { Extrakorporale Membranoxyge- } \\
\text { nierung }\end{array}$ \\
\hline EMS & „Emergency medical services" \\
\hline FFP & „Filtering face piece" \\
\hline $\mathrm{F}_{1} \mathrm{O}_{2}$ & $\begin{array}{l}\text { Inspiratorische Sauerstofffrakti- } \\
\text { on }\end{array}$ \\
\hline FORDEC & $\begin{array}{l}\text { "Facts, options, risks \& benefits, } \\
\text { decision, execution, check" }\end{array}$ \\
\hline HEMS-TC & $\begin{array}{l}\text { "Helicopter emergency medical } \\
\text { services technical crew member" }\end{array}$ \\
\hline ITH & Intensivtransporthubschrauber \\
\hline ITW & Intensivtransportwagen \\
\hline$p_{a} \mathrm{O}_{2}$ & Arterieller Sauerstoffpartialdruck \\
\hline$P C R$ & „Polymerase chain reaction“ \\
\hline PEEP & $\begin{array}{l}\text { „Positive end-expiratory } \\
\text { pressure" }\end{array}$ \\
\hline PSA & Persönliche Schutzausrüstung \\
\hline$R L$ & Rückenlage \\
\hline SARS-COV-2 & $\begin{array}{l}\text { "Severe acute respiratory } \\
\text { syndrome coronavirus } 2 "\end{array}$ \\
\hline $\mathrm{S}_{\mathrm{p}} \mathrm{O}_{2}$ & $\begin{array}{l}\text { Pulsoxymetrische Sauerstoffsät- } \\
\text { tigung }\end{array}$ \\
\hline$Z V K$ & Zentraler Venenkatheter \\
\hline
\end{tabular}


Tab. 1 Strukturierte Entscheidungsfindung mithilfe des FOR-DEC-Modells (Facts, Options, Risks \& Benefits, Decision, Execution, Check)

\begin{tabular}{|l|l|l|}
\hline Optionen & Gefahren, Risiken, Nachteile & Vorteile, Chancen \\
\hline $\begin{array}{l}\text { ITH-Transport in RL } \\
\text { ITH-Transport in BL }\end{array}$ & $\begin{array}{l}\text { Hypoxie } \\
\text { Rasche Umlagerung in RL während des Flugs } \\
\text { nicht möglich, Komplikationen durch BL }\end{array}$ & $\begin{array}{l}\text { Vertraute Situation } \\
\text { Ausreichende Oxyge- } \\
\text { nierung während des } \\
\text { Transports }\end{array}$ \\
\hline ITW-Transport in BL & $\begin{array}{l}\text { Zeitverzögerung, Verfügbarkeit? Komplikatio- } \\
\text { nen durch BL }\end{array}$ & $\begin{array}{l}\text { Ausreichende Oxyge- } \\
\text { nierung während des }\end{array}$ \\
\hline $\begin{array}{l}\text { Transports, besseres } \\
\text { Etablierung einer } \\
\text { ECMO-Therapie }\end{array}$ & $\begin{array}{l}\text { Laut Leitlinie nur bei therapierefraktärer } \\
\text { Hypoxämie indiziert, Zeitverzögerung, Ver- } \\
\text { fügbarkeit? }\end{array}$ & $\begin{array}{l}\text { Ausreichende Oxyge- } \\
\text { nierung während des }\end{array}$ \\
\hline Transportabbruch & $\begin{array}{l}\text { Fehlende Intensivbehandlungskapazität so- } \\
\text { wie keine Möglichkeit der EMCO-Therapie bei } \\
\text { weiterer Verschlechterung im abgebenden }\end{array}$ & $\begin{array}{l}\text { Transports } \\
\text { Krankenhaus }\end{array}$ \\
\hline
\end{tabular}

rige Erfahrungen im Luftrettungsdienst und Intensivtransport.

\section{Anamnese}

Die 62-jährige Patientin war am Vortag mit zunehmender Dyspnoe und $\mathrm{S}_{\mathrm{p}} \mathrm{O}_{2}$ von $65 \%$ unter Raumluft durch den Rettungsdienst in der zentralen Notaufnahme des abgebenden Krankenhauses vorgestellt worden. Seit insgesamt $4 \mathrm{Ta}$ gen habe sie über trockenen Husten und Dyspnoe geklagt. Der Ehemann der Patientin war bereits zuvor positiv auf SARS-CoV-2 getestet worden. In der bei Krankenhausaufnahme durchgeführten Röntgenthoraxaufnahme konnten schwere pneumonische Infiltrate beidseits nachgewiesen werden. Laborchemisch zeigten sich eine CRPErhöhung sowie eine Anämie und es konnte mittels PCR im Rachenabstrich eine Infektion mit SARS-CoV-2 bestätigt werden. Aufgrund der respiratorischen Insuffizienz war die Patientin zunächst spontan atmend auf die Intensivstation aufgenommen worden. Bei V.a. bakterielle Superinfektion war eine kalkulierte antiinfektive Therapie mit Piperacillin/ Tazobactam und Clarithromycin initiiert sowie ein Therapieversuch mit Hydroxychloroquin gestartet worden. Unter der Gabe von 61 Sauerstoff/min zeigte sich die respiratorische Situation anfänglich kompensiert, im weiteren Verlauf war es aber zu einer raschen Verschlechterung der Oxygenierung gekommen, sodass die Patientin noch am Abend des
Aufnahmetags intubiert und beatmet werden musste.

\section{Befund}

Unmittelbar nach Alarmierung und Durchführung des Arzt-Arzt-Gesprächs erfolgt der etwa zwanzigminütige Anflug zur abgebenden Klinik ohne Besonderheiten. Vor dem Betreten der Intensivstation legen Notarzt und HEMS-TC die gemäß den Standards des Luftrettungsbetreibers für COVID-19-Transporte erforderliche PSA an, die aus Ganzkörperoverall, 2 Paar Einmalhandschuhen, FFP-2- bzw. FFP-3-Schutzmaske und Schutzbrille besteht. Die PSA wird nach dem Vieraugenprinzip angelegt, um mögliche Leckagen oder unkorrekten Sitz zu entdecken. Im Anschluss erfolgt am Patientenbett die Übergabe der Patientin an das ITH-Team. Die Patientin ist orotracheal intubiert, invasiv beatmet und mit Sufentanil $25 \mu \mathrm{g} / \mathrm{h}$ sowie Midazolam $20 \mathrm{mg} / \mathrm{h}$ tief analgosediert. Bei schwerer Oxygenierungsstörung wurde die Patientin circa eine Stunde vor dem Eintreffen des ITH durch das Team der Intensivstation erstmalig in eine inkomplette Bauchlagerung verbracht, was zu einer Verbesserung der Oxygenierung geführt habe. Im Arzt-Arzt-Gespräch wurde allerdings im Vorfeld auf entsprechende Nachfrage kommuniziert, dass sich die Patientin in Rückenlage befinden würde. Die Patientin ist nach Intubation mit einem ZVK, einer arteriellen Kanüle, einer peripheren Ve- nenverweilkanüle, einem Blasenkatheter und einer Magensonde versorgt worden. Die Tubuslage erscheint korrekt und es gibt auskultatorisch keine Anhaltspunkte für einen Pneumothorax. Die Patientin wird mit einem Intensivrespirator invasiv druckkontrolliert beatmet $\left(\mathrm{F}_{\mathrm{I}} \mathrm{O}_{2}=0,8 ; \operatorname{PEEP} 15 \mathrm{cmH}_{2} 0\right.$, inspiratorischer Spitzendruck $32 \mathrm{cmH}_{2} 0$, Atemfrequenz 14/min, Atemminutenvolumen 7,5l/min). Die $\mathrm{S}_{\mathrm{p}} \mathrm{O}_{2}$ beträgt $93 \%$ und das endtidale $\mathrm{CO}_{2} 40 \mathrm{~mm} \mathrm{Hg}$. Die aktuelle arterielle Blutgasanalyse zeigt in inkompletter Bauchlagerung folgende Werte: $\mathrm{pH}=7,25 ; \mathrm{p}_{\mathrm{a}} \mathrm{O}_{2}=96 \mathrm{~mm} \mathrm{Hg}$, $\mathrm{p}_{\mathrm{a}} \mathrm{CO}_{2}=45 \mathrm{~mm} \mathrm{Hg}$. Der Oxygenierungsindex $\mathrm{p}_{\mathrm{a}} \mathrm{O}_{2} / \mathrm{F}_{\mathrm{I}} \mathrm{O}_{2}$ ist $120 \mathrm{~mm} \mathrm{Hg}$. Unter kontinuierlicher Applikation von Norepinephrin $0,8 \mathrm{mg} / \mathrm{h}$ ist der invasiv gemessene Blutdruck 105/50 mm Hg und die Herzfrequenz $80 / \mathrm{min}$. Die Körpertemperatur beträgt $38,3^{\circ} \mathrm{C}$.

\section{Diagnose}

- Atypische bilaterale COVID-19-

Pneumonie mit schwerem ARDS

- SARS-CoV-2-Infektion (PCR positiv)

- V.a. bakterielle Pneumonie im Sinne einer Superinfektion

- Invasive Beatmung seit dem Vortag

- Vorerkrankungen: Diabetes mellitus

Typ 2, Adipositas per magna (BMI

$41,6 \mathrm{~kg} / \mathrm{m}^{2} ; 155 \mathrm{~cm} / 99 \mathrm{~kg}$ ), arterielle Hypertonie, substituierte Hypothyreose, chronisches Schmerzsyndrom, Restless-Legs-Syndrom, Depression, chronische Hepatitis B

\section{Therapie und Verlauf}

Typischerweise werden beatmete Patienten im Intensivtransport in Rückenlage transportiert. Es wird daher mit dem Team der abgebenden Intensivstation erörtert, ob in Rückenlage ein für den Transport akzeptabler Gasaustausch zu erwarten ist. Es wird berichtet, dass sich die Patientin jeweils nach Umlagerung kurz respiratorisch verschlechtern, aber im Anschluss daran wieder ausreichend stabilisieren würde. Die Umlagerung und der anschließende ITHTransport erscheinen daher möglich, sodass die $\mathrm{F}_{\mathrm{I}} \mathrm{O}_{2}$ auf 1,0 erhöht und ein 
nichtdepolarisierendes Muskelrelaxans (Pancuronium $8 \mathrm{mg}$ ) zur Vermeidung von Asynchronität zwischen Patientin und Beatmungsgerät mit möglicher beatmungsassoziierter Lungenschädigung verabreicht wird. Im Anschluss erfolgt die Umlagerung in Rückenlage im Patientenbett. Allerdings kommt es daraufhin rasch zu einer relevanten Verschlechterung der Oxygenierung mit einem Abfall der $\mathrm{S}_{\mathrm{p}} \mathrm{O}_{2}$ auf $75 \%$. Es gibt weiterhin keine Anhaltspunkte für einen Pneumothorax oder eine inkorrekte Tubuslage. Auch endotracheales Absaugen und eine Wartezeit von 15 min führen zu keiner relevanten Verbesserung der Oxygenierung, sodass die Patientin wieder in die ursprüngliche inkomplette Bauchlagerung im Patientenbett verbracht wird. Hierunter kommt es rasch $\mathrm{zu}$ einer neuerlichen Verbesserung der $\mathrm{S}_{\mathrm{p}} \mathrm{O}_{2}$ auf Werte um $90 \%$, sodass Notarzt und HEMS-TC die Situation ohne Zeitdruck reevaluieren können.

Nach strukturierter Entscheidungsfindung wird der Entschluss getroffen, die Patientin im ITH in Bauchlagerung $\mathrm{zu}$ transportieren. Die Patientin wird daher mit insgesamt vier Personen sorgfältig in Bauchlagerung auf die Fahrtrage des ITH umgelagert und anschließend an das Transportbeatmungsgerät (HAMILTON-T1, Hamilton Medical AG, Schweiz) angeschlossen. Dies gestaltet sich problemlos, sodass schließlich 75 min nach der Ankunft auf der Intensivstation der Transport zum ITH und der anschließende Flug in die Zielklinik beginnen können (• Abb. 1). Da dort noch ein bodengebundener Zwischentransport im RTW erforderlich ist, kann die Patientin schlussendlich 50 min nach Transportbeginn ohne weitere Komplikationen in der Zielklinik übergeben werden.

Im weiteren intensivmedizinischen Verlauf stabilisierte sich die respiratorische Situation der Patientin unter intermittierender Bauchlagerung erfreulicherweise, sodass die Sedierung und die Katecholamintherapie im Verlauf ausgeschlichen werden konnten. Eine ECMO-Behandlung war nicht erforderlich geworden. Am 11. Behandlungstag erfolgte bei prolongiertem Weaning eine dilatative Punktionstracheotomie, und die Patientin konnte am 19. Behandlungstag in gebessertem Zustand druckunterstützt spontan atmend in eine Weaning-Klinik weiterverlegt werden.

\section{Diskussion}

Bei ARDS-Patienten ist die Bauchlagerung ein evidenzbasiertes Verfahren, um den pulmonalen Gasaustausch durch Veränderung des VentilationsPerfusions-Verhältnisses, durch Homogenisierung der Atemgasverteilung und durch Reduktion minder- oder nichtbelüfteter Areale mit Vergrößerung des am Gasaustausch teilnehmenden Lungenvolumens signifikant $\mathrm{zu}$ verbessern [8]. In einer prospektiven, randomisierten Multicenterstudie konnte bei beatmeten Patienten mit moderatem oder schwerem ARDS ein signifikanter Überlebensvorteil für die Bauchlagerungsgruppe gezeigt werden [4]. Die aktuelle deutsche S3-Leitlinie empfiehlt bei Patienten mit ARDS und einem Oxygenierungsindex $\mathrm{p}_{\mathrm{a}} \mathrm{O}_{2} / \mathrm{F}_{\mathrm{I}} \mathrm{O}_{2}<150 \mathrm{~mm} \mathrm{Hg}$ die Bauchlage mit einem starken Empfehlungsgrad [1]. Dagegen gibt diese Leitlinie keine Hinweise, wie bei diesen Patienten in einer Transportsituation verfahren werden soll. Die Erfahrungen hierzu sind international gering und in der Literatur sind lediglich einzelne Fallberichte verfügbar [2, 3, 5]. Die S3Leitlinie empfiehlt weiterhin den Einsatz der venovenösen ECMO bei Patienten mit schwerem ARDS nur als Rescue-Therapie bei therapierefraktärer Hypoxämie [1]. Grundsätzlich stehen extrakorporale Lungenersatzverfahren momentan nur in spezialisierten Zentren zur Verfügung. Da ein akutes Lungenversagen sehr rasch progredient verlaufen kann, ist eine frühzeitige Kontaktaufnahme mit einem ARDS-Zentrum wie im vorliegenden Fall sinnvoll, um den Einsatz eines extrakorporalen Lungenersatzverfahrens nicht zu spät in Erwägung zu ziehen. Es ist aus verschiedenen Gründen auch nicht immer möglich, eine ECMOTherapie bereits im abgebenden Krankenhaus zu initiieren. Es müssen daher regelmäßig kritisch kranke ARDS-Patienten boden- und luftgebunden verlegt werden [6].
Notfall Rettungsmed 2021 $\cdot 24: 1114-1118$ https://doi.org/10.1007/s10049-020-00798-1 (c) Springer Medizin Verlag GmbH, ein Teil von Springer Nature 2020

A. Schellhaaß · S. Pöselt · J. Schwietring · J. Horter $\cdot$ M. Münzberg

Luftgebundene Intensivverlegung in Bauchlage bei COVID-19ARDS

\section{Zusammenfassung}

Ein ITH wird für einen Transport einer Patientin mit COVID-19-ARDS disponiert. Im abgebenden Krankenhaus wird die Patientin allerdings in Bauchlagerung vorgefunden. Nach Umlagerung in Rückenlage kommt es zu einer bedrohlichen Hypoxämie, sodass die Patientin wieder in Bauchlagerung verbracht werden muss. Nach strukturierter Entscheidungsfindung kann die Patientin komplikationslos in Bauchlagerung im ITH in die Zielklinik transportiert werden. Im weiteren Verlauf stabilisiert sich die Patientin erfreulicherweise und kann druckunterstützt spontan atmend in eine Weaning-Klinik verlegt werden.

Schlüsselwörter

ARDS - Bauchlage · Patiententransport . COVID-19· Intensivtransporthubschrauber

\section{Air ambulance intensive care transport in prone position for COVID-19 ARDS}

\section{Abstract}

An EMS helicopter was scheduled to transport a woman with COVID-19 acute respiratory distress syndrome. However, the patient was found in prone position in the delivering hospital. After repositioning in the supine position, life-threatening hypoxemia occurred, so that the patient had to be returned to the prone position. After a structured decision making process, the patient could be transported with the helicopter without complications in prone position. Fortunately, the patient was stabilized in the further course of the disease and was transferred to a weaning facility breathing spontaneously with pressure support.

Keywords

ARDS - Prone position - Transportation of patients COVID-19 $\cdot$ Air ambulance 


\section{ADAC Luftrettung}

\section{Checkliste Bauchlagerung im Luftrettungsdienst}

\section{VORAB BEDENKEN}

\section{ARZT-ARZT-GESPRÄCH}

Alternativer Transport sinnvoll? Bauchlagerung notwendig? Geschlossene Absaugung installiert?

Vorsicht vor Dislokation/Diskonnektion/Abknickphänomenen Kontraindikationen vorhanden?

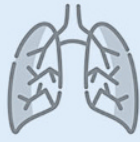

\section{INDIKATION ZUR BAUCHLAGE}

ARDS-Diagnose + Oxygenierungsindex $\mathrm{paO}_{2} / \mathrm{F}_{\mathrm{i}} \mathrm{O}_{2}<150 \mathrm{mmHg}$

schwer $\mathrm{paO}_{2} / \mathrm{F}_{\mathrm{O}_{2}} \leq 100 \mathrm{mmHg}$ bei PEEP $\geq 5 \mathrm{~cm} \mathrm{H} \mathrm{H}_{2} \mathrm{O}$

moderat $\mathrm{paO}_{2} / \mathrm{F}_{\mathrm{i}} \mathrm{O}_{2}=101-200 \mathrm{mmHg}$ bei PEEP $\geq 5 \mathrm{~cm} \mathrm{H}_{2} \mathrm{O}$ mild $\quad \mathrm{paO}_{2} / \mathrm{F}_{\mathrm{i}} \mathrm{O}_{2}=201-300 \mathrm{mmHg}$ bei PEEP $\geq 5 \mathrm{~cm} \mathrm{H}_{2} \mathrm{O}$

\section{VORSICHTSMASSNAHMEN DER HYGIENE}

Nach Handbuch Medizin Teil C

Bei COVID-19:

FFP 2+/FFP 3, Brille, Overal/Kittel, Handschuhe, Filter Dräger SafeStar55

\section{DER RICHTIGE DREH}

\section{VOR DEM DREHEN}

Absprache der Beteiligten, Beachten des Protokolls, Sonografie-Volumenstatus? Pleuraerguss? EF? Fixierung des Tubus und aller Zugänge

Erfassen der "Problembereiche" , FO, 1,0

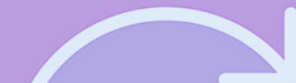

\section{WÄHREND DES DREHENS}

Ausreichende Zahl von Personen, Klare Zuordnung der Deviceüberwachung

Besondere Sicherung des Tubus

\section{NACH DEM DREHEN}

Überprüfung Tubuslage, Überprüfung aller Zugänge, Überprüfung des Beatmungsgerätes, Ggf. Absaugen der Sekrete, Monitoring etCO, Druckentlastung exponierter Stellen

\section{WICHTIG BEIM TRANSPORT}

Modell der Trage des Hubschraubers in Hinblick auf die Belastbarkeit prüfen Medizingeräte beachten
MÖGL. KONTRAINDIKATIONEN

Offenes Abdomen

Wirbelsäuleninstabilität

Erhöhter intrakranieller Druck

Bedrohliche Herzrhythmusstörungen Manifester Schock

\section{KOMPLIKATIONSMANAGEMENT}

Aufklärung der Angehörigen

Was tun bei CPR, Extubation,

Manifester Schock?

RISIKEN DES DREHENS

Ungeplante Extubation

Beatmungsprobleme

Verrutschen des Tubus

Verlust des zentralvenösen Zugangs

Lagerungsschäden

Nervenläsionen (Plexus brachialis)

\section{TRANSPORTRISIKEN}

Missgeschicke

(z.B. Dislokationen,

mangelhafte Überwachung,

Fixierung)

Inadäquate Transportbedingungen

Transportstress (Lärm, Hypothermie)

Spontanverlauf der Erkrankung

Abb. $2 \Delta$ Checkliste Bauchlagerung im Luftrettungsdienst. (Quelle: ADACLuftrettung) 
Obwohl im Vorfeld des Transports ein Arzt-Arzt-Gespräch erfolgte, wurde im abgebenden Krankenhaus eine Neubewertung der Situation erforderlich, da die Patientin zwischenzeitlich in eine inkomplette Bauchlagerung verbracht worden war und ein Transport unter den gegebenen Bedingungen in Rückenlage an der deutlich verschlechterten Oxygenierung in dieser Lagerungsposition anzunehmend gescheitert wäre. Mithilfe des aus der Luftfahrt bewährten FORDECModells („facts, options, risks \& benefits, decision, execution, check“) wurde eine strukturierte Entscheidungsfindung durchgeführt und es konnten folgende Handlungsoptionen identifiziert werden [7]:

a) Transport in Rückenlage im ITH

b) Transport in Bauchlage im ITH

c) Transport in Bauchlage im ITW

d) Hinzuziehen eines ECMO-Teams und Beginn einer ECMO-Therapie im abgebenden Krankenhaus und Transport in Rückenlage nach Stabilisierung im ITH oder ITW

e) Transportabbruch und Belassen der Patientin im abgebenden Krankenhaus

Im Anschluss wurden diese Optionen im Hinblick auf Gefahren, Risiken und Nachteile sowie Vorteile und Chancen bewertet (• Tab. 1) und es wurde entschieden, den Transport in Bauchlage im ITH durchzuführen.

Um den RTH-/ITH-Teams der ADAC Luftrettung in dieser High-risk-low-frequency-Situation eine Unterstützung im Rahmen der Entscheidungsfindung zu geben, wurde nach Auswertung der aktuellen Literatur und auf der Basis der bisher gemachten Erfahrungen zum Transport von beatmeten Patienten in Bauchlage eine Verfahrensanweisung erstellt. Das Team hat so im Vorfeld und in der konkreten Übergabesituation die Möglichkeit, anhand einer ergänzenden Checkliste alle relevanten Aspekte für eine sichere Transportdurchführung und Versorgung des Patienten zu prüfen. Die grafische Darstellung erleichtert dabei die Erfassung der wichtigsten Punkte (• Abb. 2).

In der Literatur werden folgende Komplikationen durch die Bauchlagerung beschrieben: Gesichtsödeme,
Druckulzera (Gesicht, Hornhaut, Becken, Knie, Tibiavorderkante), „Nichttoleranz"während Bauchlagerung (= Husten, Pressen, Beatmungsprobleme), Herzrhythmusstörungen, Mamillennekrosen, Tubus- oder Katheterdiskonnektionen oder -dislokationen, Abknickphänomene von Schläuchen an medizinischen Geräten und Leitungen, Nervenschäden $[1,8]$. Es ist daher bei der Umlagerung auf eine gute Fixierung des Tubus und aller Zugänge und nach Umlagerung auf eine Druckentlastung exponierter Stellen mit wenig Unterhautfettgewebe sowie die Zugänglichkeit zu allen Schläuchen und Kathetern während des gesamten Transports zu achten [6, 8]. Offenes Abdomen, Wirbelsäuleninstabilität, erhöhter intrakranieller Druck, bedrohliche Herzrhythmusstörungen und manifester Schock stellen relative Kontraindikationen zur Bauchlagerung dar, von denen bei lebensbedrohlicher respiratorischer Insuffizienz im begründeten Einzelfall allerdings abgewichen werden kann und muss $[1,8]$.

In der vorliegenden Kasuistik konnte durch die ruhige und zielgerichtete Vorgehensweise des Teams ein qualitativ hochwertiger Patiententransport in Bauchlage trotz der ausgeprägten Herausforderungen einer komplexen Beatmungssituation bei einer adipösen COVID-19-Patientin mit erforderlichem Infektionsschutz komplikationsfrei gewährleistet werden. Die konsequente Umsetzung der Hygieneschutzvorgaben mit PSA-Anlage, Einsatz eines geeigneten Beatmungsfilters und Berücksichtigung der Ablaufprozeduren hat allzeit einen unmittelbaren Zugang zur Patientin gewährleistet.

\section{Fazit für die Praxis}

- Es gibt keine ernsthaften Einschränkungen, auch einen luftgebundenen Transport eines kritisch kranken und beatmeten Patienten in Bauchlagerung durchzuführen.

- Im Vorfeld definierte Verfahrensanweisungen und Checklisten helfen eine sichere Anwendung zu ermöglichen und Komplikationen zu reduzieren.
- Alle während des Transports möglicherweise notwendigen Maßnahmen müssen nach dem Grundsatz „expect the unexpected" gut antizipiert und dem Team bekannt sein, um Komplikationen zu vermeiden, zumindest aber rasch erkennen und beheben zu können.

\section{Korrespondenzadresse}

\section{Dr. med. A. Schellhaaß, DESA}

Klinik für Anästhesie, Intensivmedizin, Schmerztherapie / OP-Abteilung, BG Klinik Ludwigshafen

Ludwig-Guttmann-Straße 13, 67071 Ludwigs-

hafen, Deutschland

Alexander.Schellhaass@bgu-ludwigshafen.de

\section{Einhaltung ethischer Richtlinien}

Interessenkonflikt. A. Schellhaaß, S. Pöselt, J. Schwietring, J. Horter und M. Münzberg geben an, dass kein Interessenkonflikt besteht.

Für diesen Beitrag wurden von den Autoren keine Studien an Menschen oder Tieren durchgeführt. Für die aufgeführten Studien gelten die jeweils dort angegebenen ethischen Richtlinien. Für Bildmaterial oder anderweitige Angaben innerhalb des Manuskripts, über die Patienten zu identifizieren sind, liegt von ihnen und/oder ihren gesetzlichen Vertretern eine schriftliche Einwilligung vor.

\section{Literatur}

1. AWMF (2017) S3-Leitlinie Invasive Beatmung und Einsatz extrakorporaler Verfahren bei akuter respiratorischer Insuffizienz. AWMF LeitlinienRegister Nr.001/021

2. DellaVolpe JD, Lovett J, Martin-Gill C et al (2016) Transport of mechanically ventilated patients in the prone position. Prehosp Emerg Care 20:643-647

3. Flabouris A, Schoettker P, Garner A (2003) ARDS with severe hypoxia-aeromedical transportation during prone ventilation. Anaesth Intensive Care 31:675-678

4. Guérin C, Reignier J, Richard JC et al (2013) Prone positioning in severe acute respiratory distress syndrome. N Engl J Med 368:2159-2168

5. Hersey D, Witter T, Kovacs G (2018) Transport of a prone position acute respiratory distress syndrome patient. Air Med J 37:206-210

6. Jahn N, Voelker MT, Bercker S et al (2017) Interhospitaltransport von Patienten mit ARDS. Anaesthesist 66:604-613

7. Soll H, Proske S, Hofinger G et al (2016) Decisionmaking tools for aeronautical teams: FOR-DEC and beyond. Aviat Psychol Appl Hum Factors 6:101-112

8. Vogt BT, Sensen B, Kluge S (2019) Bauchlagerung bei Beatmung - Schritt für Schritt. Dtsch Med Wochenschr 144:978-981 